colonization begins immediately upon birth. While adults have a gut microbiome that is quite robust and stable over time, new-borns experience a dynamic microbial colonization resulting in higher vulnerability and sensitivity to disturbances. The new-born's microbiome composition is highly dependent on delivery mode (vaginal vs. caesarean) and feeding choices (breastfeeding vs. formula), which may directly impact child's health by modulating risks for developing diseases like asthma or allergy. Breast milk is now understood to be an additional source of microbes from the mother towards the infant, and intriguingly, latest research tells that the uterus is not completely sterile, although whether it contributes to colonization prior to birth is highly controversial. In particular, the role of labour or lack thereof, remains enigmatic. This presentation will provide an overview of the different transmission routes and highlight research on different cohorts of mother-infant pairs according to different delivery modes. While many questions remain to be addressed, new insights from the scientific field may lead to new research opportunities for developing strategies that mitigate the negative effects from deviations in the early-life microbial colonization.

\section{MODE OF DELIVERY, ROUTE OF DELIVERY AND DIET ALL REGULATE INFANT MICROBIOTA AND METABOLOME}

Kieran M. Tuohy. Fondazione Edmund Mach, Italy

The intestinal microbiome is now recognised as playing an important role in human health and disease, impacting on many host physiological processes from metabolism and immune homeostasis to brain development and even cognitive function. Importantly, many of these physiological processes appear to be affected by early life events. Maternal health during pregnancy, term of birth, mode of delivery (Spontaneous Vaginally Delivered or Caesarean-section), infant diet (breast feeding, infant formula feed, fortified infant formula) and exposure to microorganisms (both pathogenic and commensal) all impact on neonatal physiological development and later life health or disease risk. Many studies, using different microbiological approaches have characterised the successional development of the infant gut microbiota. Some have also correlated microbiota composition with concomitant changes in health status (e.g. incidence of infections) or physiological biomarkers. Studies in animals and using in vitro microbiota models have identified prebiotics capable of modulating the architecture of the infant gut microbiota and intervention studies in healthy infants have also confirmed that infant formula fortified with prebiotics can modulate the gut microbiota of formula fed infants towards that of breast-fed infants. In this presentation we will assess the potential of fructans (inulin and oligofructuse in particular) as prebiotic ingredients capable of modulating the composition of the infant gut microbiota. We will discuss evidence of safety, tolerability and impact on microbiota metabolic output. Finally, we will discuss the need for wider application of whole systems metabolic profiling or metabolomics to study the metabolic consequences of microbiota modulation, using a specific example data-set. In a collaborative study with University College Cork in Ireland, we have measured the metabolic implications of early life events in terms of urinary metabolite profiles using LC-MS based metabolomics in 199 breast fed infants. Mode of delivery and term $\mathrm{v}$ pre-term birth clearly impacted on urinary metabolite profiles, with 5000 statistically significant biomarkers separating infant groups. Remarkably, these shifts in metabolite profiles reflected closely differentiative clustering of faecal microbiota at the same time point, indicating that gut microbiota derived metabolites contribute significantly to the urinary metabolome in infants and more importantly, that changes within the intestinal microbiome brought on by early life events have clear and measurable consequences in terms of infant metabolism. These observations identify metabolomics as a powerfully informative tool for studying diet:microbiota interactions, especially in infants, and a technology likely to provide new mechanistic insight linking microbiota modulation with physiological response or health effects in babies.

\section{QUALITY OF PROBIOTIC PRODUCTS: WHAT IS NEEDED? \\ H. Szajewska. The Medical University of Warsaw, Department of Paediatrics, Warsaw, Poland}

Many clinicians have concerns regarding the reliability of some of the products currently on the market. Indeed, a number of studies have questioned the microbiological quality and labeling of many commercial probiotic products. Only some of them met the definition of probiotics, i.e., contain viable, well-defined microorganisms in sufficient numbers. A substantial percentage of products contained other species of organisms. Products sold for medicinal purposes tend to be of better quality than probiotics used in dairy foods or probiotic supplements. This may be due to the fact that in most countries, probiotics are not regulated as drugs but are marketed as dietary supplements. This means that they are not standardized. However, even top quality probiotic products may suffer from some degree of product variability. Probiotics are live microorganisms that survive in the anaerobic environment. Factors such as oxygen, temperature, and humidity may have an impact on the maintaining the viability of the probiotic strains at sufficiently high levels to ensure their therapeutic activity throughout their shelf life. Additionally, variations in manufacturing processes, quality between batches, and packaging material may have an impact on the final products. Accurate labeling is essential for proper use of probiotics. Healthcare professionals and consumers should be aware of possible variations. Until issues are regulated, the only sound approach seems to be to choose probiotic products from a recognized manufacturer who has a regulated quality control of factors including the composition and content of the probiotic bacteria and adheres to good manufacturing practice. 